\title{
Grammatical mood and ambiguity aversion
}

\author{
Sacha Bourgeois-Gironde ${ }^{1}$, Alda Mari ${ }^{1}$, David Nicolas ${ }^{1}$, David Blunier ${ }^{2}$ \\ ${ }^{1}$ Institut Jean Nicod, CNRS/ENS/EHESS/PSL, France \\ ${ }^{2}$ Departement de Linguistique, Université de Genève, Switzerland \\ https://doi.org/10.36505/ExLing-2019/10/0008/000370
}

\begin{abstract}
This paper explores the impact of language on behaviour studying the impact of mood in a probabilistic choice context. Building on the idea of "aversion to ambiguity" according to which subjects prefer situations in which probabilities are known over those in which they are not known, we have systematically tested the association of sentence mood with choice situations. We found that with indicative the aversion to ambiguity is confirmed, whereas with the subjunctive, it is not. This indicates that grammatical features can influence the way in which subjects apprehend choices in probabilistic contexts.
\end{abstract}

Key words: decision theory, language, ambiguity, risk, mood, subjunctive

\section{Goal}

While modern economic theories have soundly established a connection between economic behavior and psychology, there is a new and growing interest in the connection between language and behavior.

This paper studies one aspect of this connection by addressing choices in probabilistic contexts. Specifically, we study how the verbalization of mental states accompanying choices in probabilistic contexts can modify what psychology based economic theories have labelled as the "standard" behavior. We show here that certain grammaticalized features of natural language can impact the expectations solely based on psychological considerations.

\section{Background on decision theory}

Decision theory models a relation of preference over a set of options. Typically, it models future oriented decisions, whereby different possible situations are envisaged with different probabilities of being realized. One major distinction established by economist is the one between risk and ambiguity. Consider the ballot box on the right. A situation is pictured, where there are 100 balls, 50 orange and 50 grey. The proportion between the orange and the grey balls is known. The left ballot box pictures a situation of risk, where the probabilities are known. The right ballot box represents a situation of ambiguity. The ballot box contains 100 orange and grey balls, but the proportion between the two is not known. According to the view of Savage 1954, subjects should be indifferent to this difference insofar as, subjectively, the two situations are

ExLing 2019: Proceedings of $10^{\text {th }}$ International Conference of Experimental Linguistics, 25-27 September 2019, Lisbon, Portugal 
equivalent. This, insofar as the so-called "rational" individual is considered. However, it has been widely shown since Ellsberg 1961 that individuals do not treat these decision contexts as equivalent and display what has been labeled an "aversion to ambiguity" (Epstein 2004). The preference for precise probabilistic information over ambiguous information leads to standard preferences for the former choices.

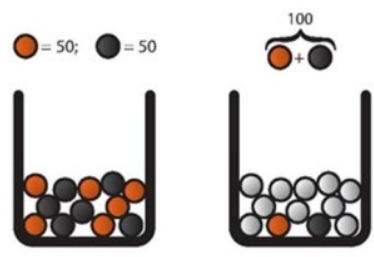

Figure 1. The urn on the left represents a risky situation; the urn on the right an ambiguity situation.

\section{The role of language and mood}

Some correlations between mood and decision behavior (investment under risk) have been probed at a macro level (Bernhofer, Constantini, Kovacic, 2018). Our study is the first to investigate how linguistic reports about choices intuitively point towards ambiguity averse or ambiguity non-averse in a stylized experimental setting.

In our study we systematically tested the association of sentence mood with choice situations and we have asked the question of whether the "standard" behavior is observed no matter what verbalization of the mental states we use. Specifically, we have studied choices for action in the context of belief predicates.

(1) I believe that it is raining.

We have used Italian, as this same English sentence can be rendered via two different grammaticalizations of mood on the verb in the embedded clause (called 'prejacent')

(2) Credo che piove.IND/piova.SUBJ -- I believe that it is raining.

In the linguistic literature on mood, there is a widespread consensus for admitting that mood is an indicator of the mental state described by the attitude, in our specific case, the belief state (Portner 2018 for an overview). Indicative is an indicator of uncertainty (this is rendered via a notion of homogeneity in the internal state (Giannakidou, Mari 2010 for a comprehensive discussion of the literature), subjunctive is an indicator of uncertainty (this is 
rendered as a non-homogeneity in the mental state). As a result, the use of subjunctive under the belief in $\backslash \operatorname{ref}\{$ subj\} indicates that there is a weakness in the mental belief state of the attitude holder that includes the possibility that both the prejacent and its negation are possible candidates for being true in the actual world. Specifically for Italian 'believe' predicate credere, different authors locate at different levels of the interpretation of the semantic component of uncertainty for belief. According to some of them (e.g. Homer 2008) belief is the weaker member of the scale <certainty, belief> and thus non-homogeneity is asserted. According to some others (Mari 2016), the relevant scale is $<$ knowledge, belief $>$. In this case, uncertainty would be coded in the presupposition with 'believe' bearing the implicated presupposition 'not know' (Mari, 2016, Giannakidou, Mari 2020). No matter whether uncertainty is located in the assertion or in the presupposition, what matters for the purpose of our experiment is that subjunctive triggers uncertainty. Our hypothesis is in (3):

(3) Different verbalizations have an impact on the 'standard' behavior $\left(\mathrm{H}_{0}\right)$

\section{Experiment}

We ran a questionnaire among native northern- and central-Italian speakers $(n=64$, ages between 18 and 50) consisting of 16 randomized items; each participant would see one condition out of four, for a total of 4 questions, corresponding to 4 different choice situations. Each participant was presented a description of a probabilistic choice situation, with two illustrations for each situation: one depicting a risk situation, the other depicting an ambiguity situation.

Each scenario was accompanied by a target sentence describing the situation. Each sentence would consist of the matrix predicate credere, and an embedded verb displaying verbal agreement with either the subjunctive or the indicative mood.

As shown, participants were asked to choose which situation the target sentence illustrated best.

\section{Results and discussion}

We found that with indicative, $\mathrm{H}_{0}$ is confirmed. With subjunctive, $\mathrm{H}_{0}$ is not confirmed (p-value: 0.007 ).

This indicates that, when the indicative is used, the default preference for the "standard" behavior for risky situations is maintained. However, this preference seems to be overridden when the subjunctive is used. 


\section{S. Bourgeois-Gironde et al.}

We can explain the results by appealing to the "weakness" of the subjunctive. As we noted above, the subjunctive triggers (a presupposition of) uncertainty. The difference between the risk ballot box and the ambiguity ballot box is that the probabilities are not known for the latter. In other terms, the ambiguity ballot box allows to accommodate uncertainty insofar as there is a parameter (the distribution of the probabilities) unknown to the subject. The subjunctive orientates the choice towards the ambiguity ballot box thus impacting on the standard behavior.

We thus acknowledge that, while the "standard" individual prefers risky situations over ambiguous ones, we see that by making explicit the states of mind that accompany a certain choice and by manipulating grammatical parameters we do no longer obtain the expected generalizations.

This result lead us to open several questions for future research; in particular, we will study whether and to what extent similar results apply to languages that do not have mood shift with belief predicates.

\section{References}

Bernhofer, J., Constantini, F., Kovacic, M. 2018. Risk attitudes, investment behavior and linguistic variation. University Ca'Foscari, Dept. of Economics Research Paper Series, 34/15.

Ellsberg, D. 1961. Risk, ambiguity, and the savage axioms. The quarterly journal of economics, 643-669.

Epstein, L.G. 2004. A definition of uncertainty aversion. In Uncertainty in Economic Theory. Routledge, 187-224.

Giannakidou, A., Mari, A. 2020. Veridicality in Grammar and Thought: modality, propositional attitudes and negation. The University of Chicago Press.

Homer, V. 2008. Intervention effects: the case of presuppositions. Master's thesis, ENS.

Mari, A. 2016. Assertability conditions of epistemic (and fictional) attitudes and mood variation. In Semantics and Linguistic Theory 26, 61-81.

Portner, P. 2018. Mood. Oxford University Press, 2018.

Savage, L.G. 1954. The foundations of statistics. Courier Corporation. 